(RESEARCH ARTICLE)

\title{
Comparative study of two isolation methods of Cyanobacteria in Côte d'Ivoire
}

\author{
Yao Rosine Djeha 1, 2,* Coulibaly Julien Kalpy 2, Koffi Mathias Ahoutou 2, Yao Eric Kouamé ${ }^{2}$, Amon Lydie ${ }^{2}$, \\ Yao René Kouamé ${ }^{1,2}$ and Djaman Joseph Allico 1,3 \\ ${ }^{1}$ Laboratory of Pharmacodynamic-Biochemistry, University Félix Houphouët-Boigny of Cocody, 22 BP 582 Abidjan 22, \\ Côte d'Ivoire.. \\ ${ }^{2}$ Department of Environment and Health / Unit of Chemistry and Environmental Microbiology, Pasteur Institute of Côte \\ d'Ivoire, BP 490 Abidjan 22, Côte d'Ivoire. \\ ${ }^{3}$ Department of Clinical and Fundamental Biochemistry, Pasteur Institute of Côte d'Ivoire, BP 490 Abidjan 22, Côte \\ d'Ivoire.
}

Publication history: Received on 04 July 2019; revised on 17 July 2019; accepted on 22 July 2019

Article DOI: https://doi.org/10.30574/gscbps.2019.8.1.0117

\begin{abstract}
Cyanobacteria are prokaryotic organisms found in the freshwaters of most countries of the world. Interest in these organisms has increased in recent years because of their ability to produce toxins that are harmful to higher organisms. The objective of this study was to compare two methods of isolation of cyanobacteria in Côte d'Ivoire. Monthly water withdrawals were carried out from June 2016 to March 2018 on the Aghien Lagoon (Côte d'Ivoire) and analyzed using two methods, namely the isolation method on modified BG11 agar and the direct binocular method. A total of 1682 cyanobacteria were isolated, 1593 (94.70\%) cyanobacteria were obtained by direct isolation and 89 $(5.30 \%)$ by seeding isolation. The 1682 cyanobacteria were purified by successive transplanting on the agar BG11 modified, 563 cyanobacteria (33.47\%) were obtained. Of this total, $92.36 \%(n=520$ cyanobacteria) were obtained by the direct method and $7.64 \%$ ( $n=43$ cyanobacteria) by the isolation method on modified BG11 agar. In order to maintain the growth of purified cyanobacteria, the 563 cyanobacteria were transferred to liquid modified BG11 medium. However 38 (6.74\%) cyanobacteria had a positive growth on this medium of which $5.76 \%(\mathrm{n}=30)$ came from the direct method and $17.02 \%(n=8)$ from the isolation method on modified BG11 agar. This study has shown that the isolation method that has resulted in better yield has been isolation by isolation method on modified BG11 agar.
\end{abstract}

Keywords: Aghien; Cyanobacteria; Isolation; Culture

\section{Introduction}

Freshwater is the main source of drinking water for most of the world's human populations [1]. Increased eutrophication of these rivers will result in massive development of phytoplankton species, mainly cyanobacteria [23].

Cyanobacteria are photosynthetic prokaryotic microorganisms naturally present in the aquatic environment (less than 20000 cells / $\mathrm{mL}$ ), without any problem being associated with it [4]. However, problems arise when there is proliferation of cyanobacteria [5]. This problem is all the more serious when the species responsible for these blooms can produce toxins. The contamination of freshwater by these toxins limits their use because they are often the cause of deadly intoxication of herds of domestic or wild animals, fish, birds and humans [6-8]. In contrast, many studies have shown that cyanobacteria are a new source of various bioactive compounds [9-10].

\footnotetext{
${ }^{*}$ Corresponding author

E-mail address: djeharosine@gmail.com
} 
Thus, for carrying out studies on their secondary metabolites, the provision of viable strains of cyanobacteria is important. This motivated the creation of numerous collections including that of the Pasteur Institute of Paris (IPP), the National Institute for Environmental Sciences (NIES) of Japan, the Institute of Hydrobiology (FACHB) of China and the University of Texas (UTEX) in the United States. However, in Africa, the collections are almost non-existent.

In Côte d'Ivoire, only studies of Coulibaly et al. [11] were conducted on the isolation and culture of cyanobacteria by testing different media, namely Conways, BG11 and Bold. Our study consisted in comparing two methods of isolation of cyanobacteria on modified BG11 medium in Côte d'Ivoire in order to set up a collection.

\section{Material and methods}

\subsection{Sampling}

Monthly water withdrawals were carried out over a 22-month period from June 2016 to March 2018 on the Aghien lagoon (Côte d'Ivoire). Samples were collected using $20 \mu \mathrm{m}$ porosity plankton net with a collector. This consisted of filtering 5 liters of surface water with the aid of the net, then recovering in the collector $100 \mathrm{ml}$ of lagoon water containing the cyanobacteria. The contents of the collector are then transferred to a one-liter bottle. This process was performed several times until the bottle was filled. The samples thus obtained were stored in a cooler containing cold accumulators and then transported to the laboratory for analysis.

\subsection{Isolation and culture of cyanobacteria}

The culture medium used for the isolation and culture of cyanobacteria was liquid and solid modified BG11 medium. These media were prepared according to the formulas and indications of Coulibaly et al. [11] at pH 7.1. Two isolation methods were performed:

\subsubsection{First method: Isolation by seeding}

$500 \mu \mathrm{L}$ of water from the lagoon were diluted successively in $1000 \mu \mathrm{l}$ of the modified liquid medium BG11, then $100 \mu \mathrm{l}$ of each dilution was spread on a Petri dish containing agar medium BG11 modified according to the described method by Rippka et al. [12].

\subsubsection{Second method: Direct isolation under a binocular loupe}

A quantity of $2 \mu \mathrm{l}$ of water from the lagoon was successively diluted in $4 \mu \mathrm{l}$ of the modified liquid medium BG11. These micro-dilutions were observed with a binocular microscope, the least concentrated was identified and cyanobacteria taken one by one using a micropipette. Each colony was transferred to a well. This process was repeated until the 96well plate, each containing $10 \mu \mathrm{l}$ of the modified BG11 liquid medium, was filled.

\subsubsection{Incubation}

The Petri dishes and the 96-well plates were sealed with Parafilm and placed in a culture room at $25 \pm 1^{\circ} \mathrm{C}$ where they were subjected to a light intensity (white light quality MAZDA FLUOR M2TF 36W / LJ $1.2 \mathrm{~m}$ long, 32 Watt) producing a daylight-like light of $175.56 \pm 1$ lux. Petri dishes and plates were observed daily with a binocular magnifying glass to monitor the growth of cyanobacteria.

\subsubsection{Successive transplanting}

After several weeks of growth, the cyanobacteria colonies on the Petri dishes were taken one by one under the binocular microscope. The agar containing the colony was cut with a scalpel blade sterilized by the Bunsen flame and transplanted streaks onto a new Petri dish.

Similarly, when the development of cyanobacteria was observed in the wells, they were collected one by one under the binocular microscope with a micropipette then transferred to a Petri dish containing the agar-modified BG11 medium.

The transplanting was repeated a ten times in order to obtain pure colonies of cyanobacteria. The cyanobacteria thus obtained were transferred into the Erlenmeyer flasks with plugs containing a volume of 10 ml of the liquid modified BG11 medium, incubated in a room at $25 \pm 1^{\circ} \mathrm{C}$ with a brightness of $175.56 \pm 1$ lux. 


\section{Results}

\subsection{Number of strains isolated according to isolation mode}

A total of 1682 cyanobacteria were isolated from the 126 lagoon water samples collected during this study. Of the total cyanobacteria obtained, $94.70 \%(n=1593)$ were isolated by the direct method and $5.30 \%(n=89)$ by the isolation method on modified BG11 agar.

\subsection{Number of cyanobacteria obtained after successive transplanting}

Of the 1682 cyanobacteria isolated a total of 563 purified cyanobacteria were obtained after repeating a few times successive transplanting on the agar medium. 520 cyanobacteria or $92.36 \%$ were obtained by the direct method and $43(7.64 \%)$ by the isolation method on modified BG11 agar.

\subsection{Number of cyanobacteria obtained after transfer to the liquid medium}

The 563 cyanobacteria obtained after the various successive subcultures were transferred to the liquid medium. After three months on the liquid medium, 38 (6.75\%) cyanobacterial strains had positive growth. Of these, 30 strains came from direct isolation at a rate of $5.76 \%$ and $8(17.02 \%)$ by isolation method on modified BG11 agar.

\subsection{Cyanobacteria rate obtained after growth according to isolated cyanobacteria}

Of a total of 1682 cyanobacteria isolated, 38 had a positive growth, a rate of $2.25 \%$ of isolated cyanobacteria. $8.98 \%(\mathrm{n}$ $=8$ ) obtained from the 89 cyanobacteria isolated by the isolation method on modified BG11 agar and $1.88 \%(\mathrm{n}=30$ ) obtained from 1593 cyanobacteria isolated by the direct isolation method.

\subsection{Cyanobacteria colonies lost during the study}

In this study, 1682 cyanobacteria were isolated by two methods. In order to obtain pure strains, successive subcultures were carried out, 563 cyanobacteria were obtained and transferred to a liquid medium, ie a $66.52 \%$ loss of cyanobacteria. 38 cyanobacteria had positive growth on this medium, a total loss of $97.74 \%$.

\section{Discussion}

Several studies have been carried out on the isolation of cyanobacteria and their toxic or beneficial effect $[13,10]$, but very few concerning exclusively isolation and culture [11]. The objective of this work was to compare two modes of isolation of cyanobacteria from the Aghien lagoon samples from June 2016 to March 2018.

A high isolation rate was observed for the direct isolation mode under a binocular loupe. This could be due to the fact that the cyanobacteria were spotted then taken one by one and put in culture. While the isolation on agar, $100 \mu \mathrm{L}$ was taken for seeding, this quantity could contain a large microbial flora and algae variety whose rapid growth would significantly prevent the isolation of cyanobacteria [14].

Thus, during the various purification tests by the successive transplanting technique, a significant loss of cyanobacteria was observed. This could be due to contamination by some bacteria or fungi in agar favored by the favorable growing conditions. Meseck et al. [15] observed a 13\% decrease in the growth rate of cyanobacteria in the presence of a bacterial community, while other authors observed total inhibition of growth [16,17]. The loss of cyanobacteria during successive transplanting could also be explained by the very loss of cyanobacteria during the transfer from one agar to another.

In this study, 38 cyanobacteria had positive growth on the medium used. Some studies have reported proportions inferior to those obtained in this study. This is the case of the studies conducted by Oufdou and Oudra [10] in Morocco which isolated and cultured 10 cyanobacteria in samples taken from 4 different sites. In France, Wilame and Hoffman [18] isolated 11 cyanobacteria in samples collected from 8 sites in the Artois-Picardie basin. However, other studies have reported higher frequencies. Berger [13] isolated 57 cyanobacteria in May 2002 samples.

This difference in result could be attributed to the mode of isolation used, to the composition of the culture medium or to the different variations of the luminous intensity of the incubation room. Indeed, all the studies cited above have used culture media different from that used in this study but an identical isolation protocol. Oufdou and Oudra [10] and [13] used the seeding method followed by the different subcultures, but different culture media, namely the liquid 
and solid media BG13 and Z8 with or without nitrogen. On the other hand, Wilame and Hoffman [18] used the BG11 culture medium supplemented with antibiotics and the seeding method followed by the different transplantation.

This growth rate of cyanobacteria on the modified BG11 medium could be explained by the development of fungi and other bacteria present in the Erlenmeyers thus contaminating the different cultures. This development of fungi and other bacteria sometimes inhibits the development of cyanobacteria. This fact was noticed by Roger and Reynaud [19] in France and [20] in Burkina Faso.

The physicochemical conditions of the culture medium could prevent the development of cyanobacteria. Thus, several authors have shown that besides the physicochemical conditions suitable for a good functioning, certain species excrete in the environment where they live toxic substances preventing the development of competing species. This was shown by Tassigny and Lefebvre [21] for non-thermal freshwater algae.

Another fact that could influence the growth of cyanobacteria is the light intensity of the growing room. For this purpose, all cyanobacteria do not develop in the same range of light intensity, some require a low intensity while others a high intensity.

\section{Conclusion}

Interest in cyanobacteria has increased in recent years given the many secondary metabolites they can synthesize. This study compared two modes of cyanobacterial isolation in Côte d'Ivoire and found that the seeding isolation method was the most conclusive. However, isolation and cultivation of cyanobacteria are difficult methods given their slow growth and rapid contamination by certain microorganisms.

\section{Compliance with ethical standards}

\section{Acknowledgments}

Authors thanks sincerely the direction of Pasteur Institute of Cote d'Ivoire, the WASAF consortium, Dr Muriel GUGGER (Pasteur Institute of Paris) and all the members of the Unit of Chemistry and Environmental Microbiology of Pasteur Institute for theirs valuables contributions and support.

\section{Disclosure of conflict of interest}

The authors declare that there are no conflicts of interest.

\section{References}

[1] Pedro O, Correia D, Lie E, Skåre JU, Leã J, Neves L, Sandvik M and Berdal K. (2011). Polymerase chain reaction (PCR) detection of the predominant microcystine producing genotype of cyanobacteria in Mozambican lakes. African Journal of Biotechnology, 10 (83), 19299-19308.

[2] Kononen K. (2001). Eutrophication, harmful algal blooms and species diversity in phytoplankton communities: Examples from the Baltic Sea. Ambio, 30 (4), 184-189.

[3] Smith VH. (2003). Eutrophication of freshwater and coastal marine ecosystems. A global problem. Environmental Science and Pollution Research, 10 (2), 126-139.

[4] Blais S. (2002). The issue of cyanobacteria (blue-green algae) at La Baie de Missisquoi in 2001. Agrosol, 13 (2), 103-110.

[5] WHO. (2003). Algae and cyanobacteria in freshwater. In: Guidelines for safe recreational water environments. Volume 1: Coastal and fresh waters. World Health Organization, Geneva, (Switzerland), 136-158.

[6] Azevedo SMFO, Carmichael WW, Jochimsen EM, Rinehart KL, Lau S, Shaw GR and Eaglesham G K. (2002). Human intoxication by microcystins during renal dialysis treatment in Caruaru. Brazil Toxicology, (181-182), 441-446.

[7] Gugger M, Molica R, Le Berre, Dufour P, Bernard C and Humbert JF. (2005). Genetic diversity of Cylindrospermopsis strains (Cyanobacteria) isolated from four continents. Applied and Environmental Microbiology, 71 (2), 1970-1100. 
[8] Van Appeldoorn ME, Van Egmond HP, Speijers GJA and Bakker GJI. (2007). Toxins of cyanobacteria. Molecules Nutrition Food Ressources, 51 (1), 7-60.

[9] Welker M and Von Döhren H. (2006). Cyanobacterial peptides. Nature's own combinatorial biosynthesis. FEMS Microbiology Revues, 30 (4), 530-563.

[10] Oufdou K and Oudra B. (2009). Bioactive substances developed by cyanobacteria isolated from certain Moroccan aquatic ecosystems. Africa Science, 5 (2), 260-279.

[11] Coulibaly Kalpy J, Groga N, Soumahoro MK, Koudougou M, Ebrottié-Brou JE, Kouassi-Haoulley MT, Ouattara A, Faye-Ketté H and DOSSO M. (2014). Preliminary trial of implementation of cyanobacteria culture in Ivory Coast. International Journal of Biological Chemical Sciences, 8 (2), 551-565.

[12] Rippka R, Waterbury J and Stanier R. (1981). Isolation and purification of cyanobacteria: Some general principles. In : Starr MP, Stops H, Triper HG, Balows A, Schelegel H G(Eds), The prokaryotes. Springer-Verlag, Berlin, 212-218.

[13] Berger C. (2005). Cyanobacteria from the lower Senegal River Delta: Diversity, toxicity, toxins and associated risks. Thesis of the National Museum of Natural History of Paris, France, 144-145.

[14] Mezouari S. (2017). Isolation and characterization of microorganisms of biotechnological interest in the region of Sidi Bel Abbes. Thesis of the Djillali Liabes University of Sidi Bel Abbes, Algeria, 35-36.

[15] Meseck SL, Smith BC, Wikfors GH, Alix JH and Kapareiko D. (2007). Nutrient interactions between phytoplankton and bacterioplankton under different carbon dioxide regimes. Journal of Applied Phycology, 19 (3), 229-237.

[16] Dakhama A, Noüe J and Lavoie M. (1993). Isolation and identification of antialgal substances produced Pseudomonas aeruginosa. Journal of Applied Phycology 5 (3), 297-306.

[17] Le Chevanton M. (2013). Microalgae-bacteria interactions in bispecific experimental system: Effects on growth of Dunaliella sp. Thesis of the University of Nantes, France, 52-53.

[18] Wilame R and Hoffman L. (2005). Cyanobacteria (blue algae) responsible for efflorescence in stagnant water. Toxicological and ecological aspects. Rapport final, 96-97.

[19] Roger PA and Reynaud PA. (1977). Methods of isolation and purification of cyanophyceae. ORSTOM Papers, Biology Series, 10 (2), 121-128.

[20] Gugger M and Couté A. (2006). Diversity and toxicity of cyanobacteria in water resources in Burkina Faso. Research Institute for Development UR167- CYROCO, 28-29.

[21] Tassigny M and Lefebvre M. (1971). Self-hetero antagonism and other consequences of excretions of water or thermal algae. International Vereinigung für Limnologie, 19, 26-38.

\section{How to cite this article}

Yao RD, Coulibaly JK, Koffi MA, Yao EK, Amon L, Yao RK and Djaman JA. (2019). Comparative study of two isolation methods of Cyanobacteria in Côte d'Ivoire. GSC Biological and Pharmaceutical Sciences, 8(1), 84-88. 